\title{
EDITORIAL
}

\section{Matemáticas con pertinencia social, cultural, y territorial}

\author{
Molly Tun ${ }^{1}$ \\ Pilar Peña Rincón ${ }^{2}$
}

Este número aporta una cantidad considerable de trabajos tanto teóricos como prácticos acerca de la Etnomatemática, y como tal, sirve como una fuente valiosa para educadores, estudiantes, y personas interesadas en el mundo diverso de las prácticas matemáticas y culturales. Los autores presentan argumentos ilustradores y originales acerca de la importancia de analizar de forma crítica las percepciones actuales que existen acerca de los límites del campo de las matemáticas. Este debate teórico responde a las consecuencias históricas de la imposición de divisiones disciplinarias occidentales, y propone fomentar una forma de pensar más inclusiva. Nos hace considerar: ¿Qué son las matemáticas? ¿Quién y cómo se define el campo? ¿Qué prácticas forman parte de esta visión de las matemáticas? ¿Quiénes realizan estas prácticas? ¿Cómo se enseñan y se transmiten estos conocimientos? Este debate no pretende definir de forma concluyente estos términos, sino promover una apertura conceptual acerca de las matemáticas que va acorde con y se nutre de la misma diversidad cultural de Latinoamérica y el sur global.

Dada la gran extensión latitudinal del continente americano, Latinoamérica como espacio geográfico representa uno de los lugares más diversos del mundo, tanto en relación con la biodiversidad, como con la diversidad humana y cultural. De hecho, no es coincidencia que la amplia variedad de ecosistemas, climas, geografías, flora, y fauna de las américas esté intrínsecamente conectada a una gama de poblaciones humanas, culturas, lenguas,

\footnotetext{
${ }^{1}$ Doctora en Literaturas y culturas hispanas y lusófonas, Universidad de Minnesota; y profesora asistente de Literatura y Cultura de Simpson College, Iowa, Estados Unidos. Email: molly.tun@simpson.edu

${ }^{2}$ Doctora en Matemática Educativa, CICATA-Instituto Politécnico Nacional de México; y jefa del Programa Pedagogía en Educación Básica de la Pontificia Universidad Católica de Chile, Santiago, Chile. Email: ppenar@uc.cl
} 
tradiciones, costumbres, prácticas, y conocimientos igual de diversos. Sin caer en el determinismo, se puede entender la conexión innata que tienen las innovaciones intelectuales, matemáticas, y culturales con sus entornos materiales, físicos, y espaciales correspondientes. En concordancia con la idea del filósofo José Ortega y Gasset "yo soy yo en mis circunstancias", las prácticas matemáticas y culturales parten de contextos específicos y propios, lo que hace imperativo nuestro trabajo etnomatemático de estudiar tal diversidad enriquecedora de conocimientos y contextos. La materialización de este esfuerzo intelectual y teórico se puede apreciar en la gran gama de espacios geográficos y contextos sociales analizados por los artículos que forman parte de este número.

Mientras los autores representados en este número promueven varios de los mismos principios de la Etnomatemática acerca de la inclusión de voces, la diversidad de perspectivas, la deliberación de modelos a seguir, y el potencial emancipatorio de estudiar y enseñar prácticas vinculadas al contexto local, cada autor opera desde su propia posicionalidad y propone diferentes herramientas para llevar a cabo tales objetivos. Este conjunto de trabajos representa una variedad de metodologías, prácticas matemáticas, y conclusiones. Una tendencia notable es el uso de entrevistas semiestructuradas, una aproximación al estudio de campo que permite a los investigadores establecer conexiones profundas y personales con los informantes, a la vez que la conversación es flexible y se gira en torno a la perspectiva y las prioridades del informante. Estas entrevistas avanzan los objetivos de la Etnomatemática al crear un contexto de análisis en el cual los diversos entrevistados (practicantes y productores) son creadores de conocimientos matemáticos. Además, es alentador ver cuántos docentes, alumnos, y ayudantes están trabajando de primera mano en las comunidades para fomentar espacios de conversación e intercambio; puesto que mientras las prácticas y productos matemáticos son aspectos culturales visibles, las perspectivas matemáticas que sostiene una sociedad sólo se pueden ver, entender, y cuestionar, a partir de conversaciones y conexiones de esta índole. También es muy valorable el tiempo y la planificación que se ha requerido para realizar tales investigaciones y reflexiones.

Abriendo el número, Julian Chamorro Becerra, Gustavo-Adolfo Marmolejo-Avenia y Edwin-Giovanni Insuasty-Portilla, presentan "Una experiencia del recorrido territorial de una 
Tun, M. \& Peña-Rincon, P. (2019). Matemáticas con pertinencia social, cultural, y territorial. Revista Latinoamericana de Etnomatemática, 12(4), 1-5. DOI: 10.22267/relatem.19124.30

etnoeducadora inga. La localización en el desarrollo del pensamiento matemático", un texto que reflexiona sobre el pensamiento matemático desarrollado en comunidades indígenas. La experiencia muestra cómo una etnoeducadora estudió el rol de la localización en procesos de espacialidad en la Institución Etnoeducativa Rural Bilingüe Iachai Wasi Carlos Tamabioy de Santiago (Valle de Sibundoy, Colombia) y logró identificar tres formas de referenciar el espacio que destacan la complejidad de la localización en condiciones socio-culturales y su inclusión en ambientes de enseñanza: literal, esquemática y auto-identificación.

En el texto "Matemáticas y educación sexual mediante modelación de ecuación de la recta", Cristian Muñoz Jeldres se enfoca en el tema de la educación sexual de Chile a nivel secundario para desarrollar actividades didácticas matemáticas de relevancia. El autor utiliza cifras de ITS en Chile para crear y analizar modelos matemáticos que se pueden incorporar al salón de clases. El trabajo también presenta información acerca de la aplicación de tales actividades que fomentan los conocimientos matemáticos, tanto como la concientización respecto a la prevención de ITS y el autocuidado.

El artículo "Una aproximación al estudio del Teorema de Pitágoras con estudiantes de secundaria" presentado por Nehemías Moreno Martínez, Marcelino Alvarado García, Rita Guadalupe Angulo Villanueva y Eduardo Carlos Briceño Solís, relata una experiencia de aula con un grupo de quince estudiantes de secundaria del estado de San Luis Potosí, México, en él se realizó una adaptación al aula de la elaboración y uso de las tolvas que se construyen en la industria de la Pailería, logrando abordar con sentido la relación matemática planteada por el Teorema de Pitágoras.

En el trabajo "Procesos de medición en una práctica artesanal del caribe colombiano. Un estudio desde la Etnomatemática" los autores Camilo Andrés Rodríguez-Nieto, Armando Aroca Araújo, y Flor Monserrat Rodríguez-Vásquez describen, de forma detallada, el proceso de elaboración de bollos de yuca. Esta práctica artesanal no sólo es gastronómica sino también matemática y se basa en múltiples formas de medir, contar, y calcular. Además de presentar los datos recolectados durante su estadía con un comerciante reconocido de Sibarco, Colombia, los autores presentan las medidas, las equivalencias, y las fórmulas numéricas que informan el proceso de forma accesible para uso en el contexto escolar. 
A continuación, la investigación por Roberta Luzia Soares de Souza y Lucélida de Fátima Maia da Costa titulada "Ideias matemáticas na prática de um serralheiro: contexto para o ensino de matemática" describe las prácticas numéricas, trigonométricas, y geométricas de un herrero con años de experiencia en la profesión que trabaja en la ciudad de ParintinsAmazonas. El objetivo del estudio es proveer un contexto que puede ser usado por maestros y profesores para desarrollar y enseñar ideas matemáticas más relevantes y significativas, de acuerdo al entorno de sus alumnos. Otro resultado de tal estudio es fomentar una apreciación al valor intelectual de las prácticas matemáticas, como éstas, que existen a nuestro alrededor pero que no siempre se han estudiado.

Lucas Silva Pires y Doriane dos Santos Feitosa colaboraron con alumnos de la Escola Boa Esperança de Vila Tauari en Brasil para crear contenido sociocultural y matemático para el uso curricular en el contexto de la educación rural. Su artículo "Função do $1^{\circ}$ Grau em Narrativas de productores de leite bovino" es el producto de extensas conversaciones con productores de leche bovina, recolección de datos, y aplicación de resultados en el contexto escolar, específicamente a la enseñanza de funciones del primer grado. Esta investigación contribuye al programa Etnomatemática al promover la interpretación de situaciones reales, la valorización de saberes de la vida cotidiana, y la apropiación de saberes matemáticos.

En el artículo "Etnomatemática e Trabalho Colaborativo na Educação Inclusiva de Adultos no Contexto da Economia Solidária" Renata Cristina Geromel Meneghetti y Edinei de Oliveira Filho presentan las actividades pedagógicas que planearon y realizaron en el contexto de un Emprendimiento Económico Solidario de la ciudad de São Carlos. En este caso, la Economía Solidaria promueve alternativas económicas con el fin de incluir los que han sido excluidos por el sistema económico capitalista; las etnomatemáticas se usan para imaginar formas de calcular y relacionarse basadas en la solidaridad y la cooperación en vez del capitalismo. Los autores evidencian cómo las lecciones matemáticas pueden llevar a transformaciones sociales, así resaltando la importancia de las etnomatemáticas.

Por último, Marcelo Bergamini Campos, nos presenta el libro "A educação matemática no contexto da economia solidária" coordinado por Renata Cristina Geromel Meneghetti. El libro expone presenta una investigación-acción sobre el proceso de enseñanza y aprendizaje 
Tun, M. \& Peña-Rincon, P. (2019). Matemáticas con pertinencia social, cultural, y territorial. Revista Latinoamericana de Etnomatemática, 12(4), 1-5. DOI: 10.22267/relatem.19124.30

de las Matemáticas mediante intervenciones pedagógicas realizadas con los integrantes de tres empresas económicas solidarias, que buscan contribuir a que los participantes asuman una postura emancipadora en el entorno en el que se insertan. El estudio toma como referentes teóricos el Programa de Etnomatemática y la Resolución de Problemas para discutir las Matemáticas de manera significativa y contextualizada.

De este modo, el conjunto de artículos presentados en este número, contribuyen a problematizar los límites del campo de las matemáticas y de su enseñanza, a partir de experiencias didácticas concretas fundadas en una perspectiva etnomatemática. Todas ellas tienen en común que logran enseñar matemáticas con sentido y con pertinencia social, cultural y/o territorial. Esperamos que lo disfruten. 